\title{
DIVERSIDAD AGRONÓMICA DE POBLACIONES DE JITOMATE TIPO "CHERRY" S. lycopersicum L. Y S. pimpinellifolium L. CON POTENCIAL EN EL MEJORAMIENTO GENÉTICO
}

\author{
AGRONOMIC DIVERSITY OF "CHERRY" TYPE TOMATO POPULATIONS S. lycopersicum \\ L. AND S. pimpinellifolium L. WITH POTENTIAL IN PLANT BREEDING
}

\author{
Lilia Salgado-Meraz ${ }^{1}$, Ricardo Lobato-Ortiz ${ }^{*}$ Laura J. Pérez-Flores ${ }^{3}$, \\ Serafín Cruz-Izquierdo ${ }^{1}$, Cecilia Peña-Valdivia ${ }^{2}$ y J. Jesús García-Zavala'
}

\begin{abstract}
${ }^{1}$ Colegio de Postgraduados, Posgrado en Recursos Genéticos y Productividad-Genética, ${ }^{2}$ Postgrado de Botánica. Campus Montecillo, Texcoco, Edo de México, México. ${ }^{3}$ Universidad Autónoma Metropolitana Iztapalapa, Departamento de Ciencias de la Salud, CDMX, México.
\end{abstract}

*Autor para correspondencia (rlobato@colpos.mx)

\section{RESUMEN}

La variabilidad genética de los cultivares modernos de jitomate (Solanum lycopersicum L.) es reducida, por lo que los fitomejoradores requieren explorar germoplasma nativo de jitomate y de parientes silvestres para localizar genes con valor agronómico y comercial. El objetivo de este estudio fue evaluar la diversidad agronómica de cuatro poblaciones nativas de $S$. lycopersicum premejoradas y de nueve silvestres de S. pimpinellifolium, junto con un testigo comercial tipo "cherry", para identificar germoplasma sobresaliente. En dos ciclos de cultivo en invernadero se registraron 13 variables agronómicas, en un diseño de bloques completos al azar, con tres repeticiones y unidades experimentales de diez macetas con una planta por maceta. Entre poblaciones hubo significancia en todas las variables. La población nativa CP14063 fue sobresaliente, pues igualó estadísticamente al híbrido testigo en peso promedio de fruto y lo superó (12\%) en rendimiento (REN). La población CP14065 sobresalió en contenido de sólidos solubles (SST) e índice de sabor (IS), con 9.1 y 16.6. Las poblaciones silvestres LA1576, LA1584 y LA0373 también sobresalieron en SST con valores de $9.6,9.0$ y 8.3. Por lo anterior esas poblaciones son valiosas como fuente de alelos para el mejoramiento genético de la calidad del jitomate. El análisis de conglomerados formó cuatro grupos, que permitieron diferenciar a las poblaciones nativas de las silvestres y del híbrido por sus características agronómicas. La caracterización de poblaciones nativas y silvestres de jitomate permitió detectar las sobresalientes con potencial para incorporarlas a programas de mejoramiento genético.

Palabras clave: Solanum lycopersicum, S. pimpinellifolium, mejoramiento genético, poblaciones nativas, recursos genéticos.

\section{SUMMARY}

The genetic variability of the modern cultivars of tomato (Solanum lycopersicum L.) is reduced, then breeders need to explore native germplasm of tomato and wild relatives to locate genes with agronomic and commercial value. The objective of this study was to evaluate the agricultural diversity of four native populations of $S$. lycopersicum with pre-breeding and nine wild accessions of S. pimpinellifolium, along with a commercial "cherry" type as check, to identify the outstanding materials. Thirteen agronomic traits were evaluated during two cycles of cultivation in a greenhouse using a complete randomized block design, with three replications and experimental units of ten pots with a plant per pot. There was significance for all the variables among populations. Native population CP14063 was outstanding, since it matched statistically to the hybrid control on average fruit weight and yield exceeded by $12 \%$. The population CP 14065 excelled in soluble solids content (TSS) and taste (IS) with 9.1 and 16.6. Wild populations LA1576, LA1584 and LA0373 excelled too, since they obtained TSS values of 9.6, 9.0 and 8.3, respectively. Therefore, they are valuable as source of alleles for the genetic improvement of the tomato quality. Cluster analysis formed four groups, which allowed separating the native populations from the wild ones and the hybrid by their agronomic characteristics. The characterization of native and wild populations of tomatoes allowed to identify the outstanding ones with potential to be incorporated into tomato breeding programs.

Index words: Solanum lycopersicum, S. pimpinellifolium, genetic improvement, native populations, genetic resources.

\section{INTRODUCCIÓN}

El jitomate (Solanum lycopersicum L.) es la hortaliza más popular y aceptada en la cultura gastronómica del mundo, pues sus frutos se consumen frescos o industrializados. El jitomate se cultiva en más de cien países; de ellos China, EE.UU., India y Turquía producen $57 \%$ del total, y México ocupa el décimo lugar en su producción (FAO, 2017).

La domesticación del jitomate provocó cambios en su anatomía y en su forma de reproducción, pasando de alógama a autógama. La mayoría de estos cambios se atribuyen a la selección en pocos loci (Nesbitt y Tanksley, 2002); además, su reproducción por autofecundación provocó que el jitomate cultivado muestre variación genética escasa (Rick y Fobes, 1975; Miller y Tanksley, 1990), por lo que las variedades nuevas generalmente provienen de la progenie de híbridos genéticamente relacionados (Tanksley y Mc Couch, 1997).

El mejoramiento genético del jitomate requiere diversidad alélica o base genética amplia. Con la reducción de la variabilidad, el potencial para el mejoramiento a largo plazo está en peligro (Tanksley y Mc Couch, 1997), y por lo general, las características agronómicas de mayor interés para el mejoramiento genético a menudo se heredan 
cuantitativamente y se originan en poblaciones naturales (Fridman et al., 2000). Comúnmente, la diversidad genética se concentra en centros de origen y diversidad donde aún se usan y conservan variedades nativas y silvestres (Mercer y Perales, 2010), que no son objeto de mejoramiento formal (Bellon et al., 2003). Para jitomate, México es centro de domesticación y diversidad (Rick, 1976; Peralta y Spooner, 2007), por lo que cuenta con una gran variabilidad morfológica y genética en esta especie (Martínez-Vázquez et al., 2017).

Los objetivos de los programas de mejoramiento del jitomate incluyen obtener variedades con rendimientos mayores, con frutos de vida de anaquel y calidad nutricional mayores y también mejor sabor (Bai y Lindhout, 2007). Un objetivo principal es incrementar el contenido de sólidos solubles totales (SST), por su correlación alta con el sabor (Liu et al., 2007). Mediante selección asistida por marcadores se han logrado resultados positivos, pero aún falta incrementar la diversidad genética (Lecomte et al., 2004); por lo que, para mejorar el SST también podría recurrirse al aprovechamiento de rasgos genéticos de especies silvestres, como Solanum pimpinellifolium, Solanum pennellii, Solanum cheesmaniae y Solanum chmielewskii (Grandillo y Tanksley, 1996).

En México, dado el alto costo de la semilla comercial de jitomate producida por empresas extranjeras y la escasez de investigación pública en esta especie, es conveniente generar programas nacionales de mejoramiento genético y utilizar el potencial de los parientes silvestres y variedades nativas, para lo cual es necesario colectarlo, conservarlo, caracterizarlo y evaluarlo (Bonilla-Barrientos et al.,
2014; Flores-Hernández et al., 2017).

El objetivo de esta investigación fue caracterizar la diversidad agronómica de poblaciones de jitomate nativas premejoradas y silvestres para identificar materiales sobresalientes en variables agronómicas y de calidad, para proponerlos como fuente de germoplasma en el mejoramiento genético.

\section{MATERIALES Y MÉTODOS}

\section{Material genético}

Se evaluaron 14 materiales genéticos: cuatro poblaciones nativas tipo cherry, nueve poblaciones de la especie $S$. pimpinellifolium y el híbrido comercial "sweet heart" tipo Cherry de la compañía Sakata (Cuadro 1). Los jitomates nativos se colectaron directamente en campos de cultivo; sus semillas se sembraron y cultivaron en invernadero, se seleccionaron las plantas más vigorosas y sus semillas se emplearon en este estudio. Las semillas de S. pimpinellifolium las donó el Tomato Genetics Resource Center (TGRC), de la Universidad de California, Davis, EE.UU. El estudio se realizó en los ciclos de cultivo primavera-verano (P-V) 2015 y P-V 2016, en un invernadero en Montecillo, Texcoco, Estado de México (19³0' N, $98^{\circ} 53^{\prime}$ O y altitud de 2250 metros sobre el nivel del mar).

\section{Diseño experimental y manejo agronómico}

Los 14 tratamientos, constituidos por las 13 poblaciones más el híbrido comercial, fueron evaluados en un diseño experimental de bloques completos al azar con tres

Cuadro 1. Identificación, etiqueta, características y especies de Solanum evaluadas.

\begin{tabular}{lccc}
\hline Identificación & Material & Características & Especie \\
\hline 1 & LA1547 & Población silvestre & S. pimpinellifolium \\
2 & LA1602 & Población silvestre & S. pimpinellifolium \\
3 & LA0442 & Población silvestre & S. pimpinellifolium \\
4 & LA0373 & Población silvestre & S. pimpinellifolium \\
5 & LA1237 & Población silvestre & S. pimpinellifolium \\
6 & LA1576 & Población silvestre & S. pimpinellifolium \\
7 & LA1584 & Población silvestre & S. pimpinellifolium \\
8 & LA1593 & Población silvestre & S. pimpinellifolium \\
9 & LA1689 & Población silvestre & S. pimpinellifolium \\
10 & CP14060 & Población nativa cultivada & S. lycopersicum \\
11 & CP14062 & Población nativa cultivada & S. Iycopersicum \\
12 & CP14063 & Población nativa cultivada & S. Iycopersicum \\
13 & CP14065 & Población nativa cultivada & S. lycopersicum \\
14 & Sweet Heart & Cherry comercial & S. lycopersicum \\
\hline
\end{tabular}


repeticiones y 10 plantas por repetición. La siembra se hizo en charolas de unicel de 200 cavidades de $3.1 \times 3.1 \mathrm{~cm}$, con sustrato peat moss. En ambos ciclos, el trasplante se efectuó 30 días después de la siembra (dds) en bolsas de polietileno color negro, de $40 \times 40 \mathrm{~cm}$, en las que se usó arenilla de tezontle rojo como sustrato con granulometría de $\sim 4 \mathrm{~mm}$. Las plantas se regaron con solución nutritiva (Steiner, 1984), con concentración inicial de 50 \% durante la etapa vegetativa y al $100 \%$ durante floración y fructificación. El pH de la solución se mantuvo entre 5.5 y 6.0. Las plantas se mantuvieron con tutores de rafia y se podaron para mantener un solo tallo por planta. En forma preventiva se aplicaron Captan ${ }^{\circledR}$ Cupravit@ y Previcur ${ }^{\circledR}$ contra tizón tardío (Phytophthora infestans), Confidor ${ }^{\circledR}$, Beleaf ${ }^{\circledR}$ y Ampligo ${ }^{\circledR}$ contra paratrioza (Bactericera cockerelli) y mosca blanca (Bemisia tabaci Gennadius), con las dosis recomendadas por los fabricantes.

\section{Variables registradas}

Se registraron 13 variables, ocho de ellas de acuerdo con el Manual de Descriptores del Tomate (Solanum lycopersicum), del International Plant Genetic Resources Institute (IPGRI, 1996), siendo: altura de planta a los 95 dds (ALT, en $\mathrm{cm})$, altura del primer racimo (ARU, en $\mathrm{cm})$, días para la maduración del fruto del primer racimo (DM), número de flores del tercer racimo (NF3R), número total de racimos por planta (NTR), número total de frutos (NTF), peso total de frutos (REN, en g por planta) y peso promedio del fruto (PPF, en g). Las variables NTF y REN corresponden a la suma de dos cortes de fruto que se hicieron durante los ciclos de producción.

Para evaluar la forma del fruto (FOR) se calculó el cociente del diámetro polar y el transversal de los frutos (Grandillo y Tanksley, 1996). La firmeza del fruto (FIR) se midió con un texturómetro TA.XT2i, con punta cilíndrica de $0.2 \mathrm{~mm}$. El SST se midió con un refractómetro digital (PAL$1 \circledR$; Tokio, Japón) con amplitud de 0.0 a 53.0. La acidez titulable (AT) se evalúo de acuerdo con el procedimiento de la Association Official Methods of Analysis Chemists (AOAC, 2000) y se expresó como porcentaje de ácido cítrico. El índice de sabor (IS) se calculó con el cociente de SST y AT. Para medir el diámetro polar, la longitudinal del fruto y las variables PPF, FIR, SST y AT se tomaron diez frutos en madurez de consumo del tercer y cuarto racimo.

\section{Análisis estadístico}

Los datos de ambos ciclos se analizaron mediante un análisis de varianza combinado usando el modelo lineal del diseño de bloques completos al azar con factores de variación Ambientes y la interacción genotipos x ambientes. También se hizo una comparación múltiple de medias entre tipos de poblaciones y entre poblaciones con la prueba de Tukey $(P \leq 0.05)$ con el paquete estadístico SAS Institute (2002). Finalmente, mediante el paquete cluster 2.0.6 del software R.3.4.1. y las 13 variables se realizó un análisis de conglomerados mediante la distancia euclidiana y el método de agrupación UPGMA, donde la altura de corte se determinó de acuerdo con Charrad et al. (2014).

\section{RESULTADOS Y DISCUSIÓN}

El análisis de varianza detectó diferencias significativas $(P \leq 0.01)$ entre ciclos en todas las variables, excepto en FOR (Cuadro 2 y 3). Estos resultados son similares a los de Hernández-Bautista et al. (2014), quienes al evaluar en tres ciclos de producción una población nativa (S. lycopersicum L.), una silvestre (S. pimpinellifolium) y su cruza, encontraron significancia para la fuente de variación ciclos en las variables NTF, NF3R y DM, resultados atribuibles a que las condiciones de evaluación fueron distintas a través de ciclos, lo cual se evidenció por la significancia de las variables estudiadas.

También se encontraron diferencias para todas las variables en las fuentes de variación Poblaciones y en su partición en Nativas y Silvestres (Cuadro 2 y 3). Esto es evidencia de la amplia variación natural entre los dos tipos de poblaciones y dentro de cada grupo de materiales, pertenecientes a diferentes especies (Fernie et al., 2006). Esa variabilidad es susceptible de utilizarse en programas de mejoramiento genético (Dumas et al., 2003) ya que ambas especies son compatibles y producen progenie viable cuando se cruzan tanto en generaciones F1 como en generaciones avanzadas (Hernández-Bautista et al., 2016a, b).

La interacción población $x$ ciclo resultó significativa para todas las variables, excepto para FOR, NTR y PPF. Esto indica que para las variables con significancia, los genotipos expresaron promedios diferentes a través de los ciclos de evaluación, como respuesta del genotipo a los efectos cambiantes del ambiente, ya que estos no fueron los mismos, como quedó de manifiesto por la significancia entre ciclos. Esto implica que se tiene diversidad genética amplia en los materiales evaluados, cuya respuesta a los efectos del ambiente no fue la misma a través de ambientes (ciclos). Sólo en algunas variables como la forma del fruto, número total de racimos y peso promedio de fruto los promedios no cambiaron a través de los ciclos de evaluación (Cuadro 4).

En general, las poblaciones nativas fueron sobresalientes en DM, NF3R, SST e IS, mientras que las poblaciones silvestres tuvieron los valores mayores en NTR, NTF, SST, AT e IS, lo cual se observa generalmente en las poblaciones silvestres (Lecomte et al., 2004). La contribución de 
Cuadro 2. Cuadrados medios y coeficientes de variación de siete características agronómicas en poblaciones nativas y silvestres y un híbrido comercial cultivados en los ciclos primavera-verano de 2015 y 2016 en Montecillo, México.

\begin{tabular}{lrrrrrrrr}
\hline F.V. & $\mathrm{GL}$ & \multicolumn{1}{c}{$\mathrm{REN}$} & $\mathrm{DM}$ & \multicolumn{1}{c}{ NF3R } & \multicolumn{1}{c}{ ALT } & NTR & \multicolumn{1}{c}{ ARU } & NTF \\
\hline Ciclo & 1 & $28,823.05 * *$ & $3268.76 * *$ & $987.43 * *$ & $8907.44 * *$ & $51.86 * *$ & $352.19 * *$ & $37002.01 * *$ \\
Poblaciones & 13 & $3,588,382.15 * *$ & $253.04 * *$ & $1363.93 * *$ & $3672.71 * *$ & $8.66 * *$ & $584.07 * *$ & $126309.67 * *$ \\
$\quad$ Nativo & 3 & $2,270,765.67 * *$ & $194.60 * *$ & $2358.38 * *$ & $2351.11 * *$ & $4.38 * *$ & $511.44 * *$ & $10614.04 * *$ \\
$\quad$ Silvestres & 8 & $281,231.46 * *$ & $209.00 * *$ & $851.21 * *$ & $583.87 * *$ & $5.30 * *$ & $290.63 * *$ & $156759.58 * *$ \\
$\quad$ Nat vs Sil & 1 & $26,989,204.15 * *$ & $175.02 \mathrm{~ns}$ & $161.30 \mathrm{~ns}$ & $3526.59 * *$ & $56.70 * *$ & $3360.82 * *$ & $193851.70 * *$ \\
$\quad$ Híb vs Nat & 1 & $1,184,848.13 * *$ & $500.21 * *$ & $3729.68 * *$ & $2960.13 *$ & $4.56 *$ & $13.33 \mathrm{~ns}$ & $44121.00 * *$ \\
Pob *ciclo & 13 & $126,796.25 * *$ & $64.68 * *$ & $248.76 * *$ & $757.00 * *$ & $0.34 \mathrm{~ns}$ & $123.91 * *$ & $8912.65 * *$ \\
Error & 52 & $4228.28 * *$ & $1.68 \mathrm{~ns}$ & $8.58 \mathrm{~ns}$ & $142.10 \mathrm{~ns}$ & $0.84 \mathrm{~ns}$ & $2.71 \mathrm{~ns}$ & $518.30 \mathrm{~ns}$ \\
C.V. & & 7.32 & 1.29 & 8.05 & 5.49 & 8.73. & 8.00 & 8.88 \\
\hline
\end{tabular}

Nat vs Sil: nativos vs silvestres, Híb vs Nat: híbrido vs nativo, Pob: población, GL: grados de libertad, **P $\leq 0.01$, *P $\leq 0.05$, ns: no significativo, REN: peso total de frutos (g por planta), DM: días a madurez, NF3R: número de flores del tercer racimo, ALT: altura de planta a los 95 dds (cm), NTR: número total de racimos, ARU: altura al primer racimo (cm), NTF: número total de frutos.

Cuadro 3. Cuadrados medios y coeficientes de variación en seis características del fruto en poblaciones nativas y silvestres y un híbrido comercial cultivados en los ciclos primavera-verano de 2015 y 2016 en Montecillo, México.

\begin{tabular}{lrccccccr}
\hline F.V. & GL & PPF & SST & FIR & FOR & AT & IS \\
\hline Ciclo & 1 & $6.24 *$ & $37.73 * *$ & $0.254 * *$ & 0.004 & $\mathrm{~ns}$ & $0.049 * *$ & $192.80 * *$ \\
Poblaciones & 13 & $531.21 * *$ & $7.28 * *$ & $0.186 * *$ & 0.080 ** & $2.080 * *$ & $56.89 * *$ \\
$\quad$ Nativo & 3 & $335.11 * *$ & $8.76 * *$ & $0.020 * *$ & 0.080 ** & $0.070 * *$ & $54.20 * *$ \\
$\quad$ Silvestres & 8 & $0.60 * *$ & $7.26 * *$ & $0.250 * *$ & $0.150 * *$ & $0.140 * *$ & $5399.00 * *$ \\
$\quad$ Nat vs Sil & 1 & $3287.92 * *$ & $0.96 \mathrm{~ns}$ & $0.290 *$ & $0.570 * *$ & $0.630 * *$ & $143.73 * *$ \\
$\quad$ Híb vs Nat & 1 & $679.25 * *$ & $6.08 *$ & $0.120 * *$ & $0.340 * *$ & $0.007 \mathrm{~ns}$ & $11.56 \mathrm{~ns}$ \\
Pob *ciclo & 13 & $1.07 \mathrm{~ns}$ & $2.51 * *$ & $0.121 * *$ & 0.001 ns & $0.400 * *$ & $14.89 * *$ \\
Error & 52 & $1.29 \mathrm{~ns}$ & $0.10 \mathrm{~ns}$ & $0.007 \mathrm{~ns}$ & $0.002 \mathrm{~ns}$ & $0.003 \mathrm{~ns}$ & $0.40 \mathrm{~ns}$ \\
C.V. & & 14.06 & 4.21 & 7.965 & 4.280 & & 14.120 & 5.60
\end{tabular}

Nat vs Sil: nativos vs silvestres, Híb vs Nat: híbrido vs nativo, Pob: población, GL =grados de libertad. $\star \star P \leq 0.01, \star P \leq 0.05$, ns: no significativo, PPF: peso promedio del fruto (g), SST: contenido de sólidos solubles, FIR firmeza del fruto ( $\mathrm{kg} \mathrm{m} \mathrm{s}^{-2}$ ), FOR: forma, AT: acidez titulable ( \% de ácido cítrico) e IS: índice de sabor

poblaciones silvestres en el mejoramiento de variedades comerciales tipo saladette y tipo bola ha sido limitado, al anteponer como principales objetivos del mejoramiento al rendimiento y tamaño de fruto; sin embargo, S. pimpinellifolium posee alelos que incrementan SST y el tamaño de fruto, los cuales se expresan cuando son transferidos a variedades con fruto de tamaño mayor (Hernández-Bautista et al., 2014). Por lo anterior, estas se pueden usar sin ningún problema para el mejoramiento de variedades tipo cherry o con fruto grande, haciendo uso de retrocruzas y selección clásica. Además, la tecnología de los marcadores moleculares, específicamente el mejoramiento asistido de marcadores moleculares (MAS) y particularmente la retrocruza asistida por marcadores moleculares (MAB), puede minimizar los problemas asociados a caracteres indeseables incorporados durante el proceso de mejoramiento con parientes silvestres o nativos criollos, fenómeno que es conocido como "linkage drag" o arrastre por ligamiento (Tanksley y Mc Couch, 1997).
El análisis por población (Cuadro 5) indicó que CP14063 fue la de rendimiento mayor, con $2453 \mathrm{~g}$ por planta, inclusive estadísticamente superior al híbrido testigo en $12 \%$. Las poblaciones más tardías fueron el híbrido testigo, LA1547 y CP14063, con más de 100 días para maduración. Por otro lado, CP14062 tuvo el mayor NF3R (63), le siguió LA1576 (54) y el testigo fue uno de los menores (13). Esto puede ser una ventaja para los materiales experimentales de este estudio, ya que existe una correlación positiva entre el número de flores por racimo con el SST (Hernández-Bautista et al., 2014). Las poblaciones LA1602 y LA1576 mostraron NTF mayor (589 y 509) que el resto de las poblaciones silvestres, las nativas y el testigo. Al respecto, a pesar de la estrecha relación genética se puede observar que estas especies difieren en aspectos morfológicos, muchos de los cuales son heredados poligénicamente (Grandillo y Tanksley, 1996; Fridman et al., 2000). 
Cuadro 4. Comparación de medias de 13 características agronómicas de planta y fruto en poblaciones nativas y silvestres y un híbrido comercial de Solanum evaluadas en los ciclos primavera-verano de 2015 y 2016 en Montecillo, México.

\begin{tabular}{lcccc}
\hline Variables & Poblaciones nativas & Poblaciones silvestres & Híbrido & DSH (0.05) \\
\hline REN (g por planta) & $1672.04 \mathrm{~b}$ & $397.47 \mathrm{c}$ & $2168.83 \mathrm{a}$ & 247.60 \\
DM (días) & $101.22 \mathrm{ab}$ & $97.95 \mathrm{~b}$ & $111.43 \mathrm{a}$ & 10.66 \\
ALT (cm) & $185.50 \mathrm{c}$ & $231.99 \mathrm{a}$ & $210.40 \mathrm{~b}$ & 20.76 \\
ARU (cm) & $29.73 \mathrm{a}$ & $15.50 \mathrm{~b}$ & $28.25 \mathrm{a}$ & 4.30 \\
NTR & $9.04 \mathrm{~b}$ & $10.96 \mathrm{~b} \mathrm{a}$ & $10.32 \mathrm{ab}$ & 1.40 \\
NF3R & $40.27 \mathrm{a}$ & $37.18 \mathrm{a}$ & $12.18 \mathrm{~b}$ & 4.76 \\
NTF & $193.61 \mathrm{~b}$ & $301.63 \mathrm{a}$ & $97.74 \mathrm{c}$ & 40.33 \\
PPF (g) & $16.28 \mathrm{~b}$ & $2.22 \mathrm{c}$ & $28.17 \mathrm{a}$ & 1.91 \\
SST & $7.42 \mathrm{ab}$ & $7.66 \mathrm{a}$ & $6.29 \mathrm{~b}$ & 1.24 \\
FIR $\left(\mathrm{kg} \mathrm{m} \mathrm{s}^{-2}\right)$ & $0.92 \mathrm{~b}$ & $1.05 \mathrm{a}$ & $1.08 \mathrm{a}$ & 0.12 \\
FOR & $0.86 \mathrm{c}$ & $1.04 \mathrm{~b}$ & $1.12 \mathrm{a}$ & 0.02 \\
AT $(\%)$ & $0.59 \mathrm{~b}$ & $0.78 \mathrm{a}$ & $0.55 \mathrm{~b}$ & 0.08 \\
IS & $13.29 \mathrm{a}$ & $10.35 \mathrm{a}$ & $11.74 \mathrm{a}$ & 3.38 \\
\hline
\end{tabular}

Medias en fila con la misma letra son estadísticamente iguales (Tukey, 0.05). REN: peso total de frutos, DM: días a maduración del fruto del primer racimo, ALT: altura de planta a los 95 dds, ARU: altura al primer racimo, NTR: número total de racimos, NF3R: número de flores del tercer racimo, NTF: número total de frutos, PPF: peso promedio del fruto, SST: contenido de sólidos solubles, FIR firmeza del fruto, FOR: forma, AT: acidez titulable e IS: índice de sabor. DSH: Diferencia significativa honesta.

Cuadro 5. Comparación de medias de siete características agronómicas de 13 poblaciones nativas y silvestres de $z$ evaluadas en los ciclos primavera-verano de 2015 y 2016 en Montecillo, México.

\begin{tabular}{lccccccc}
\hline Material genético & $\begin{array}{c}\text { REN } \\
\text { (gpor planta) }\end{array}$ & DM (días) & NF3R & NTR & NTF & ALT (cm) & ARU (cm) \\
\hline CP14060 & $1289 \mathrm{~d}$ & $96.3 \mathrm{~d}$ & $15 \mathrm{e}$ & $10.2 \mathrm{~b}-\mathrm{f}$ & $152 \mathrm{i}$ & $163 \mathrm{~g}$ & $32 \mathrm{bc}$ \\
CP14062 & $1860 \mathrm{c}$ & $99.5 \mathrm{c}$ & $63 \mathrm{a}$ & $9.7 \mathrm{c}-\mathrm{f}$ & $250 \mathrm{ef}$ & $175 \mathrm{~g}$ & $18 \mathrm{ef}$ \\
CP14063 & $2453 \mathrm{a}$ & $109.5 \mathrm{a}$ & $39 \mathrm{c}$ & $8.3 \mathrm{f}$ & $173 \mathrm{hi}$ & $203 \mathrm{ef}$ & $40 \mathrm{a}$ \\
CP14065 & $1086 \mathrm{e}$ & $99.8 \mathrm{c}$ & $44 \mathrm{c}$ & $8.7 \mathrm{ef}$ & $200 \mathrm{gh}$ & $202 \mathrm{f}$ & $29 \mathrm{~cd}$ \\
LA 0442 & $467 \mathrm{~g}$ & $95.8 \mathrm{de}$ & $27 \mathrm{~d}$ & $11.8 \mathrm{ab}$ & $243 \mathrm{e}-\mathrm{g}$ & $220 \mathrm{c}-\mathrm{f}$ & $13 \mathrm{gh}$ \\
LA 1547 & $364 \mathrm{gh}$ & $109.5 \mathrm{a}$ & $44 \mathrm{c}$ & $10.5 \mathrm{a}-\mathrm{e}$ & $342 \mathrm{c}$ & $244 \mathrm{ab}$ & $33 \mathrm{~b}$ \\
LA 1602 & $752 \mathrm{f}$ & $92.5 \mathrm{f}$ & $52 \mathrm{~b}$ & $12.2 \mathrm{a}$ & $589 \mathrm{a}$ & $228 \mathrm{a}-\mathrm{d}$ & $11 \mathrm{~h}$ \\
LA0373 & $394 \mathrm{gh}$ & $93.7 \mathrm{ef}$ & $41 \mathrm{c}$ & $11.8 \mathrm{ab}$ & $281 \mathrm{de}$ & $248 \mathrm{a}$ & $12 \mathrm{gh}$ \\
LA1237 & $424 \mathrm{~g}$ & $95.7 \mathrm{de}$ & $40 \mathrm{c}$ & $11.3 \mathrm{a}-\mathrm{c}$ & $303 \mathrm{~cd}$ & $223 \mathrm{~b}-\mathrm{f}$ & $12 \mathrm{gh}$ \\
LA1576 & $660 \mathrm{f}$ & $94.5 \mathrm{~d}-\mathrm{f}$ & $54 \mathrm{~b}$ & $10.7 \mathrm{a}-\mathrm{d}$ & $509 \mathrm{~b}$ & $237 \mathrm{a}-\mathrm{c}$ & $13 \mathrm{gh}$ \\
LA1584 & $163 \mathrm{ij}$ & $104.3 \mathrm{~b}$ & $30 \mathrm{~d}$ & $10.2 \mathrm{~b}-\mathrm{f}$ & $146 \mathrm{i}$ & $225 \mathrm{a}-\mathrm{f}$ & $15 \mathrm{fg}$ \\
LA1593 & $281 \mathrm{hi}$ & $94.2 \mathrm{~d}-\mathrm{f}$ & $30 \mathrm{~d}$ & $11.7 \mathrm{ab}$ & $215 \mathrm{f}-\mathrm{h}$ & $227 \mathrm{a}-\mathrm{e}$ & $13 \mathrm{gh}$ \\
LA1689 & $73 \mathrm{j}$ & $102.5 \mathrm{~b}$ & $18 \mathrm{e}$ & $9.3 \mathrm{~d}-\mathrm{f}$ & $88 \mathrm{j}$ & $237 \mathrm{a}-\mathrm{c}$ & $19 \mathrm{e}$ \\
Híbrido & $2169 \mathrm{~b}$ & $111.5 \mathrm{a}$ & $13 \mathrm{e}$ & $10.3 \mathrm{a}-\mathrm{e}$ & $98 \mathrm{j}$ & $211 \mathrm{~d}-\mathrm{f}$ & $28 \mathrm{~d}$ \\
DSH (0.05) & 132 & 2.6 & 3.0 & 1.9 & 46.2 & 24.2 & 3 \\
\hline
\end{tabular}

Medias en columnas con la misma letra son estadísticamente iguales (Tukey, 0.05). REN: peso total de frutos, DM: Días a maduración del fruto del primer racimo, NF3R: número de flores del tercer racimo, NTR: número total de racimos, NTF: número total de frutos, ALT: altura de planta a los 95 dds, ARU: altura al primer racimo, DSH: Diferencia significativa honesta. 
Con respecto a las características de calidad (Cuadro 6), la población nativa CP14065 y las silvestres LA1576 y LA1584 presentaron el contenido mayor de SST (9.0 a 9.6), estadísticamente iguales entre sí y superiores al resto de las poblaciones y al testigo comercial. Esto puede deberse a que las poblaciones nativas y silvestres de jitomate tienen potencial genético para mayor producción y acumulación de SST en el fruto, y muchas de ellas producen frutos con concentraciones superiores a las de variedades comerciales cultivadas (Fridman et al., 2000; Carrari y Fernie, 2006). Los resultados fueron similares a los que tuvieron algunas de las poblaciones evaluadas por Juárez-López et al. (2009). Estos autores evaluaron siete poblaciones nativas con SST de entre 5.8 y 8.0. Parra-Gómez et al. (2016) evaluaron 225 líneas S3 tipo cherry, con variación de SST entre 3.4 y 10.4 en las mejores líneas. Gaspar-Peralta et al. (2012) reportaron SST de 3.9 a 5.2 en un grupo de ocho líneas avanzadas, derivadas de poblaciones nativas del tipo "riñón" y "calabaza".

Las diferencias en FIR (de 1.10 a 1.25) entre las poblaciones LA0442, LA1547, LA1602, LA0373, LA1576 y LA1593 no resultaron estadísticamente diferentes. Esos valores fueron inferiores a los obtenidos por Bonilla-Barrientos et al. (2014) en frutos nativos de Puebla y Oaxaca, con FIR de hasta 1.78. Al respecto, el control genético de la firmeza es complejo y depende del genotipo y de interacciones genético-ambientales, lo que indica que la firmeza del fruto se afecta con las condiciones ambientales, como el año de cultivo y la localidad (Chaib et al., 2007).

La población LA1689 tuvo el valor de AT más alto (1.0 $\%)$, el valor menor $(0.5 \%)$ correspondió a las poblaciones CP14060, CP14063, CP14065 y el híbrido tuvo $0.6 \%$. El valor más alto de IS fue de las poblaciones LA1576 y CP14065 (17.4 y 16.6), en tanto que el menor correspondió a LA1547 (7.4). Características como SST, AT y el cociente SST/AT, conocido como IS, mostraron estar entre los principales factores determinantes en la preferencia de sabor del jitomate (Causse et al., 2002). Existe una correlación positiva y significativa entre la aceptabilidad y el SST, pero negativa para ciertos niveles de ácido; sin embargo existe un nivel óptimo en el IS y la aceptabilidad (Baldwin et al., 1998). De acuerdo con Beckles (2012), valores de SST inferiores a 5, AT de 0.4 e IS de 12.5 indican un buen sabor. Además, Suarez et al. (2008) señalaron que un IS inferior a 7 indica un sabor pobre. Todas las poblaciones en el presente estudio presentaron AT superior a 0.4 y SST mayores a 5; pero, con base en IS las mejores poblaciones fueron CP14063, CP14060, CP14065 y LA1476, al superar el valor de 12.5 .

En el dendrograma generado con el análisis de conglomerados, los materiales genéticos evaluados formaron cuatro grupos (Figura 1). El grupo I estuvo formado por la población CP14063 y el híbrido testigo, que tienen similitudes en REN, SST y PPF. Estos resultados concuerdan con la correlación negativa entre rendimiento, tamaño y SST

Cuadro 6. Comparación de medias de seis características de fruto de 13 poblaciones de jitomate nativo y silvestre evaluadas en los ciclos primavera-verano de 2015 y 2016 en Montecillo, México.

\begin{tabular}{lclcllr}
\hline Material genético & PPF $(\mathrm{g})$ & SST & FIR $\left(\mathrm{kg} \mathrm{m} \mathrm{s}^{-2}\right)$ & FOR & AT & IS \\
\hline CP14060 & $13 \mathrm{bc}$ & $7.5 \mathrm{~cd}$ & $0.90 \mathrm{ef}$ & $1.20 \mathrm{~b}$ & $0.53 \mathrm{f}$ & $14.3 \mathrm{~b}$ \\
CP14062 & $14 \mathrm{~b}$ & $7.0 \mathrm{de}$ & $0.90 \mathrm{ef}$ & $0.98 \mathrm{c}-\mathrm{e}$ & $0.74 \mathrm{~d}$ & $9.5 \mathrm{~d}$ \\
CP14063 & $27 \mathrm{a}$ & $6.2 \mathrm{~g}$ & $0.87 \mathrm{ef}$ & $1.33 \mathrm{a}$ & $0.52 \mathrm{f}$ & $12.8 \mathrm{c}$ \\
CP14065 & $11 \mathrm{c}$ & $9.1 \mathrm{a}$ & $1.01 \mathrm{c}-\mathrm{e}$ & $1.25 \mathrm{ab}$ & $0.55 \mathrm{f}$ & $16.6 \mathrm{a}$ \\
LA 0442 & $3 \mathrm{~d}$ & $6.8 \mathrm{ef}$ & $1.25 \mathrm{a}$ & $0.90 \mathrm{e}$ & $0.69 \mathrm{de}$ & $9.9 \mathrm{~d}$ \\
LA 1547 & $2 \mathrm{~d}$ & $6.6 \mathrm{e}-\mathrm{g}$ & $1.15 \mathrm{a}-\mathrm{c}$ & $0.97 \mathrm{c}-\mathrm{e}$ & $0.91 \mathrm{ab}$ & $7.4 \mathrm{f}$ \\
LA 1602 & $2 \mathrm{~d}$ & $7.1 \mathrm{de}$ & $1.16 \mathrm{a}-\mathrm{c}$ & $1.00 \mathrm{~cd}$ & $0.73 \mathrm{~d}$ & $9.6 \mathrm{~d}$ \\
LA0373 & $2 \mathrm{~d}$ & $8.3 \mathrm{~b}$ & $1.23 \mathrm{ab}$ & $0.90 \mathrm{e}$ & $0.86 \mathrm{bc}$ & $9.8 \mathrm{~d}$ \\
LA1237 & $2 \mathrm{~d}$ & $7.1 \mathrm{de}$ & $0.95 \mathrm{de}$ & $0.97 \mathrm{c}-\mathrm{e}$ & $0.60 \mathrm{ef}$ & $12.3 \mathrm{c}$ \\
LA1576 & $2 \mathrm{~d}$ & $9.6 \mathrm{a}$ & $1.18 \mathrm{ab}$ & $1.05 \mathrm{c}$ & $0.56 \mathrm{f}$ & $17.4 \mathrm{a}$ \\
LA1584 & $2 \mathrm{~d}$ & $9.0 \mathrm{a}$ & $0.74 \mathrm{fg}$ & $0.93 \mathrm{de}$ & $0.93 \mathrm{ab}$ & $10.0 \mathrm{~d}$ \\
LA1593 & $2 \mathrm{~d}$ & $6.7 \mathrm{e}-\mathrm{g}$ & $1.10 \mathrm{a}-\mathrm{d}$ & $0.97 \mathrm{c}-\mathrm{e}$ & $0.77 \mathrm{~cd}$ & $8.9 \mathrm{de}$ \\
LA1689 & $2 \mathrm{~d}$ & $7.7 \mathrm{bc}$ & $0.71 \mathrm{~g}$ & $1.02 \mathrm{~cd}$ & $0.99 \mathrm{a}$ & $7.8 \mathrm{ef}$ \\
Híbrido & $28 \mathrm{a}$ & $6.3 \mathrm{fg}$ & $1.08 \mathrm{~b}-\mathrm{d}$ & $0.90 \mathrm{e}$ & $0.55 \mathrm{f}$ & $11.7 \mathrm{c}$ \\
DSH (0.05) & 2.31 & 0.64 & 0.16 & 0.09 & 0.11 & 1.3 \\
\hline
\end{tabular}

Medias en columnas con la misma letra son estadísticamente iguales (Tukey, 0.05). PPF: peso promedio del fruto, SST: contenido de sólidos solubles, FIR: firmeza del fruto, FOR: forma, AT: acidez titulable e IS: índice de sabor, DSH: Diferencia significativa honesta. 


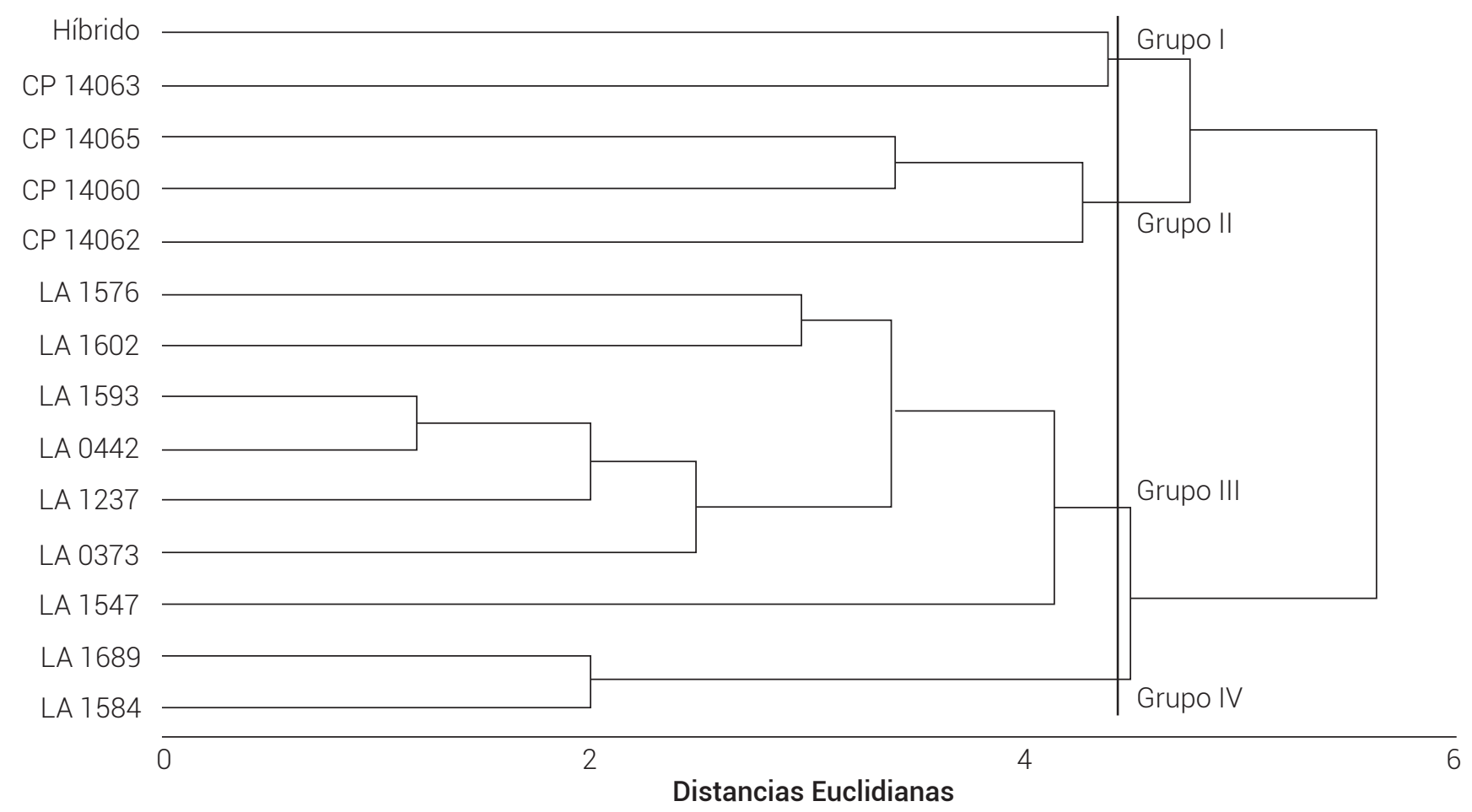

Figura 1. Dendrograma de 13 poblaciones nativas y silvestres y un híbrido comercial, con base en 13 variables agronómicas evaluadas en los ciclos primavera-verano de 2015 y 2016 en Montecillo, México.

(Bai y Lindhout, 2007), debido a que los materiales presentaron el rendimiento mayor y los valores menores de SST.

El grupo II lo formaron las poblaciones CP14062, CP14060 y CP14065; las tres poblaciones son nativas y tuvieron similitudes en DM, NTR, PPF y FIR.

Las poblaciones silvestres, a pesar de presentar gran diversidad, incluso mayor que la observada en otras especies (Flores-Hernández et al., 2017), formaron dos grupos. El grupo III concentró las poblaciones LA1593, LA0442, LA1237, LA0373, LA1602, LA1576 y LA1547. Este grupo fue similar en características como DM, NR, ARU y REN. El grupo IV se formó con las poblaciones LA1584, y LA1689, que tuvieron similitudes en las variables NR y AT.

Las diferencias entre los grupos con las poblaciones silvestres indicaron que el grupo III fue el más precoz (DM) y con valores mayores de ARU y REN. En general, las poblaciones silvestres presentaron rendimientos inferiores comparados con las poblaciones nativas y el híbrido comercial; sin embargo, tuvieron mayor número de frutos, debido al valor adaptativo que presentan sus alelos en el ámbito de selección natural silvestre, pues los frutos pequeños tienen una ventaja en la dispersión; además, producen una mayor cantidad de semillas (Tanksley, 2004).

\section{CONCLUSIONES}

Se identificaron materiales de jitomate con características sobresalientes de rendimiento, sólidos solubles totales e índice de sabor que podrían utilizarse en programas de mejoramiento genético como nueva fuente de variación genética. La población silvestre LA1576 y las poblaciones nativas CP14065, CP14060 y CP14063 tuvieron valores superiores a 12.8 de IS, que indica un buen sabor.

De las poblaciones nativas, CP14063 destacó en rendimiento (2463 g), superando a todas las poblaciones y al híbrido testigo en $12 \%$, con SST aceptable de $6^{\circ} \mathrm{Bx}$, por lo que ésta podría usarse directamente para siembras comerciales o como germoplasma promisorio para el mejoramiento genético, mientras que CP14065 sobresalió por su alto contenido de SST (9.1).

Las poblaciones silvestres LA1576 y LA1584 alcanzaron valores de 9.6 y $9.0^{\circ} \mathrm{Bx}$, respectivamente, por lo que dichas poblaciones podrían emplearse como fuente de germoplasma en programas de mejoramiento de jitomate para esta característica. 


\section{BIBLIOGRAFÍA}

AOAC, Association of Official Analytical Chemists (2000) Official Methods of Analysis. $16^{\text {th }}$ Edition USA. $1298 \mathrm{p}$

Bai Y. and P. Lindhout (2007) Domestication and breeding of tomatoes: what have we gained and what can we gain in the future? Annals of Botany 100:1085-1094. https://doi.org/10.1093/aob/ mcm 150 .

Baldwin E. A., J. W. Scott, M. A. Einstein, T. M. M. Malundo, B. T. Carr, R. L. Shewfelt and K. S. Tandon (1998) Relationship between sensory and instrumental analysis for tomato flavor. Journal of the American Society for Horticultural Science. 123:906-915. https:// onlinelibrary.wiley.com/doi/abs/10.1111/j.1365-2621.1985. tb10452.x

Beckles D.M. (2012) Factors affecting the postharvest soluble solids and sugar content of tomato. Postharvest Biology and Technology. 63:129-140. https://doi: 10.1016/j.postharvbio.2011.05.016.

Bellon M. R., J. Berthaud, M. Smale, J. A. Aguirre, S. Taba, F. Aragón, J. Díaz and $\mathrm{H}$. Castro (2003) Participatory landrace selection for on farm conservation: an example from the Central Valleys of Oaxaca, Mexico. Genetic Resources and Crop Evolution 50:401-416. https://doi.: 10.1023/A:1023967611495.

Bonilla-Barrientos 0., R. Lobato-Ortiz, J. J. García-Zavala, S. Cruz-Izquierdo, D. Reyes-López, E. Hernández-Leal y A. Hernández-Bautista (2014) Diversidad agronómica y morfológica de tomates arriñonados y tipo pimiento de uso local en Puebla y Oaxaca, México. Revista Fitotecnia Mexicana 37:129-139. https://www.revistafitotecniamexicana.org/documentos/37-2/4r.pdf.

Carrari F, and A. R. Fernie (2006) Metabolic regulation underlying tomato fruit development. Journal of Experimental Botany 57:18831897. https://doi.org/10.1093/jxb/erj020

Causse M., V. Saliba-Colombani, L. Lecomte, P. Duffe, P. Rousselle and M. Buret (2002) QTL analysis of fruit quality in fresh market tomato: a few chromosome regions control the variation of sensory and instrumental traits. Journal of Experimental Botany 53:20892098. http://www.jstor.org/stable/23697406.

Chaib J., M. F. Devaux, M. G. Grotte, K. Robini, M. Causse, M. Lahaye and I. Marty (2007) Physiological relationships among physical, sensory, and morphological attributes of texture in tomato fruits. Journal of Experimental Botany 58:1915-1925. doi:10.1093/ $\mathrm{jxb} / \mathrm{erm046}$

Charrad M, N. Ghazzali, V. Boiteau and A. Niknafs (2014) NbClust: An R Package for Determining the Relevant Number of Clusters in a Data Set. Journal of Statistical Software 61:1-36. https://doi: 10.18637/jss.v061.i06.

Dumas Y., M. Dadomo, G. Di Lucca and P. Grolier (2003) Effects of environmental factors and agricultural techniques on antioxidant content of tomatoes. Journal of the Science Food and Agriculture 83:369-382. https://doi: 10.1002/jsfa.1370.

FAO, Food Agricultural Organization (2017) Organización de las Naciones Unidas para la Agricultura y la Alimentación. Disponible en: http://www.fao.org/faostat/en/\#data/QC/visualize (Julio 2017)

Fernie R. A., Y. Tadmor and D. Zamir (2006) Natural genetic variation for improving crop quality. Current Opinion in Plant Biology 9:196202. https://doi 10.1016/j.pbi.2006.01.010

Flores-Hernández L. A., R. Lobato-Ortiz, J. J. García-Zavala, J. D. MolinaGalán, D. M. Sargerman-Jarquín y M. J. Velasco-Alvarado (2017) Parientes silvestres del tomate como fuente de germoplasma para el mejoramiento genético de la especie. Revista Fitotecnia Mexicana 40:83-91. https://www.revistafitotecniamexicana. org/documentos/40-1/9a.pdf

Fridman E., T. Pleban and D. Zamir (2000) A recombination hotspot delimits a wild-species quantitative trait locus for tomato sugar content to 484bp within an invertase gene. Proceedings of the National Academy of Sciences of the United State of America 97:4718-4723. https://doi.org/10.1073/pnas.97.9.4718.

Gaspar-Peralta P., J. C. Carrillo-Rodríguez, J. L. Chávez-Servia, A. M. VeraGuzmán e I. Pérez-León (2012) Variación de caracteres agronómicos y licopeno en líneas avanzadas de tomate (Solanum Lycopersicum L.) Phyton 81: 15-22 http://www.scielo.org.ar/scielo. php?script=sci_arttext\&pid=S1851-56572012000100003.

Grandillo S. and S. D. Tanksley (1996) QTL analysis of horticultural traits differentiating the cultivated tomato from the closely related species Lycopersicon pimpinellifolium. Theoretical Applied Genetics 92:935-951. http://doi: 10.1007/BF00224033.

Hernández-Bautista A., R. Lobato-Ortiz, S. Cruz-Izquierdo, J. J. García-Zavala, J. L. Chávez-Servia (2014) Variación fenotípica, heterosis y heredabilidad de una cruza interespecífica de jitomate. Interciencia 39:327-332. http://www.redalyc.org/html/339/33930879011/.

Hernández-Bautista A., R. Lobato-Ortiz, J. J. García-Zavala, F. López-Fortoso, S. Cruz- Izquierdo, J. L. Chávez-Servia and M. Cadeza-Espinosa (2016a) Quantitative trait locus mapping associated with earliness and fruit weight in tomato. Scientia Agricola 73:478-486. http://dx.doi.org/10.1590/0103-9016-2015-0245.

Hernández-Bautista A., R. Lobato-Ortiz, J. J. García-Zavala, M. A. ParraGómez, M. Cadeza-Espinosa, D. Canela-Doñan ... and J. L. ChávezServia (2016b) Implications of genomic selection for obtaining F2:3 families of tomato. Scientia Horticulturae 207:7-13. DOI: 10.1016/j.scienta.2016.05.005.

IPGRI, Instituto Internacional de Recursos Fitogenéticos (1996) Descriptores de Tomate (Lycopersicon spp.). Ed. Instituto Internacional de Recursos Fitogenéticos, Roma Italia. $49 \mathrm{p}$.

Juárez-López P., R. Castro-Brindis, T. Colinas-León, P. Ramírez-Vallejo, M. Sandoval-Villa, D. W. Reed, ... y S. King (2009) Evaluación de calidad de frutos de siete genotipos nativos de jitomate (Lycopersicon esculentum var. cerasiforme). Revista Chapingo, Serie Horticultura 15:5-9. http://www.scielo.org.mx/scielo.php?script=sci_ arttext\&pid=S1027-152X2009000400002.

Lecomte L., A. Gautier, A. Luciani, P. Duffé, F. Hospital, M. Buret and M. Causse (2004) Recent advances in molecular breeding: the example of tomato breeding for flavor traits. Acta Horticulturae 637:231242. https://doi.org/10.17660/ActaHortic.2004.637.28.

Liu H. F., M. Genard, S. Guichard and N. Bertin (2007) Model-assisted analysis of tomato fruit growth in relation to carbon and water fluxes. Journal of Experimental Botany 58:3567-3580. https:// doi.org/10.1093/jxb/erm202.

Martínez-Vázquez E. de los A, A. Hernández-Bautista, R. Lobato-Ortiz, J. J. García-Zavala y D. Reyes-López (2017) Exploring the breeding potential of Mexican tomato landraces. Scientia Horticulturae 220:317-325. https://doi: 10.1016/j.scienta.2017.03.031

Mercer K. L. and H. R. Perales (2010) Evolutionary response of landraces to climate change in centers of crop diversity. Evolutionary Applications 3: 480-493. https://doi.org/10.1111/j.17524571.2010.00137.x.

Miller J. C. and S. D. Tanksley (1990) RFLP analysis of phylogenetic relationships and genetic variation in the genus Lycopersicon Theoretical and Applied Genetics 80:437-448. https://doi: 10.1007/BF00226743.

Nesbitt T. C. and S. D. Tanksley (2002) Comparative sequencing in the genus Lycopersicon: implications for the evolution of fruit size in the domestication of cultivated tomatoes. Genetics 162: 365379. https://www.ncbi.nlm.nih.gov/pubmed/12242247.

Parra-Gómez M. A., R. Lobato-Ortiz, J. J. García-Zavala, D. Reyes-López y M. J. Velasco-Alvarado (2016) Evaluación de líneas de una cruza interespecífica de tomate. Revista Fitotecnia Mexicana 39:5965. http://www.scielo.org.mx/scielo.php?script=sci_arttext\&pi $\mathrm{d}=\mathrm{S} 0187-73802016000100010$.

Peralta I. E. and D. M. Spooner (2007) History, Origin and Early Cultivation of Tomato (Solanaceae). In: Genetic Improvement of Solanaceous Crop, Vol. 2: Tomato. M. K. Razdan and A. K. Mattoo (eds) Science Publishers. Enfield, New Hampshire, USA. pp: 1-24.

Rick C. M. (1976) Tomato, Lycopersicon esculentum (Solanaceae). In: Evolution of Crop Plants, N. W. Simmonds (ed). Ed. Longman Group. London. pp: 268-273.

Rick C. M. and J. F. Fobes (1975) Allozyme variation in the cultivated tomato and closely related species. Bulletin of the Torrey Botanical Club 102:376-384. https://link.springer.com/article/10.1007/BF00272889.

SAS Institute (2002) User's Guide of SAS (Statistical Analysis System). SAS Institute Inc. Cary, N. C. USA. 550 p.

Steiner A. A. (1984) The Universal Nutrient Solution. In: Proceedings 6th International Congress on Soilless Culture. Wageningen, The Netherlands. pp: 633-650

Suarez M. H., E. M. R. Rodriguez, C. D. Romero (2008) Chemical composition of tomato (Lycopersicon esculentum) from Tenerife, the 
Canary Islands. Food Chemical 106:1046-1056. https://doi: 10.1016/j.foodchem.2007.07.025

Tanksley S. D. (2004) The genetic developmental and molecular bases of fruit size and shape variation in tomato. Plant Cell 16: S181-
S189. https://doi.org/10.1105/tpc.018119

Tanksley S. D. and S. R. Mc Couch (1997) Seed banks and molecular maps: unlocking genetic potential from the wild. Science 277: 10631066. https:// doi.org/10.1126/science.277.5329.1063 
